\title{
The clinical value of valve metagenomic next-generation sequencing when applied to the etiological diagnosis of infective endocarditis
}

\author{
Sishi Cai ${ }^{1} \wedge$, Ye Yang ${ }^{2}$, Jue Pan ${ }^{1}$, Qing Miao ${ }^{1}$, Wenting Jin ${ }^{1}$, Yuyan Ma ${ }^{1}$, Chunmei Zhou ${ }^{3}$, Xiaodong Gao ${ }^{4}$ \\ Chunsheng Wang ${ }^{2}$, Bijie $\mathrm{Hu}^{1,4}$
}

${ }^{1}$ Department of Infectious Diseases, Zhongshan Hospital, Fudan University, Shanghai, China; ${ }^{2}$ Department of Cardiac Surgery, Zhongshan Hospital, Fudan University, Shanghai, China; ${ }^{3}$ Department of Microbiology, Zhongshan Hospital, Fudan University, Shanghai, China; ${ }^{4}$ Department of Hospital Infection Management, Zhongshan Hospital, Fudan University, Shanghai, China

Contributions: (I) Conception and design: S Cai, B Hu, C Wang; (II) Administrative support: B Hu, J Pan, X Gao; (III) Provision of study materials or patients: S Cai, Y Yang, C Zhou; (IV) Collection and assembly of data: S Cai, W Jin, Y Ma; (V) Data analysis and interpretation: S Cai, Q Miao; (VI) Manuscript writing: All authors; (VII) Final approval of manuscript: All authors.

Correspondence to: Chunsheng Wang. Department of Cardiac Surgery, Zhongshan Hospital, Fudan University, 180 Fenglin Road, Shanghai 200032, China. Email: wang.chunsheng@zs-hospital.sh.cn; Bijie Hu. Department of Infectious Diseases, Department of Hospital Infection Management, Zhongshan Hospital, Fudan University, 180 Fenglin Road, Shanghai 200032, China. Email: hu.bijie@zs-hospital.sh.cn.

Background: Metagenomic next-generation sequencing (mNGS) is widely applied in the etiological diagnosis of infectious diseases. However, the clinical practice of mNGS in infective endocarditis (IE) is relatively less studied. This research aimed to assess the etiological diagnostic value of valve mNGS in IE.

Methods: We retrospectively analyzed 49 IE patients who underwent cardiac valve surgery in Zhongshan Hospital, Fudan University, Shanghai from 1 June 2018 to 30 November 2020. Among these IE patients, 28 were culture positive and 21 were culture negative. The culture results of the culture-positive IE patients were set as gold standard to assess the sensitivity and specificity of valve mNGS in the etiological diagnosis of IE. We studied the positive detection rate of pathogens by valve mNGS among the culture-negative IE patients. During the same period, we also collected the resected valves of 8 patients with non-infective valvular diseases for mNGS as negative controls.

Results: The valve mNGS results of the culture-positive IE patients were the exact same as their culture results. Both the sensitivity and specificity of valve mNGS were $100 \%$. The positive detection rate of pathogens by valve mNGS was $100 \%$ among the culture-negative IE patients. The stringent mapped reads number of genera (SMRNG), relative abundance of genera, stringent mapped reads number of species (SMRN), relative abundance of species, and coverage rate of valve mNGS results were significantly higher in culture-positive IE participants than in culture-negative IE participants. The valve mNGS results of the 8 participants with non-infective valvular diseases were all negative.

Conclusions: Valve mNGS is a promising technology for the etiological diagnosis of IE, especially culture-negative IE, and it may be used to guide precise antibiotic treatment after surgery.

Keywords: Metagenomic next-generation sequencing (mNGS); etiological diagnosis; infective endocarditis (IE); culture negative infective endocarditis

Submitted Apr 29, 2021. Accepted for publication Jun 04, 2021.

doi: $10.21037 / \mathrm{atm}-21-2488$

View this article at: http://dx.doi.org/10.21037/atm-21-2488

\footnotetext{
^ ORCID: 0000-0002-7983-9436.
} 


\section{Introduction}

Infective endocarditis (IE) is a severe disease with high mortality, which can be life-threatening without proper treatment (1-3). The annual incidence of IE is estimated to be 15 to 80 cases per million persons. For high-risk population of IE such as patients with valve prostheses or with prior IE, the annual incidence of IE enormously rises to 4-6 cases per 1,000 persons or $>10$ cases per 1,000 persons (2). The main indications for valve surgery in IE include heart failure, uncontrolled infection and prevention of embolism (1). The poor prognosis of IE justifies any attempt to improve accurate diagnosis and therapeutic management. Rapid and precise identification of the causative pathogen is crucial to the etiological diagnosis and optimal antimicrobial treatment of IE. Conventional methods to detect pathogens of IE mainly rely on blood culture and vegetation culture which have low sensitivity, especially in patients with previous antibiotic treatment and IE related to fastidious or unculturable microbes. According to previous research, blood culture negative infective endocarditis (BCNE) accounts for $2.1-35 \%$ of all IE cases, and remains a diagnostic and therapeutic challenge for clinicians (4).

Fastidious or non-culturable microbes are important causes of BCNE, such as HACEK group bacteria (5), Abiotrophia spp., Bartonella spp. (6), Tropheryma whipplei (7), Coxiella burnetii, Mycoplasma spp., and fungi (8). These microbes require specific culture media and their growth is slow. Previous research has mainly recommended serological tests and 16S rRNA polymerase chain reaction (PCR) to detect pathogens in BCNE, which has outperformed conventional culture under certain circumstances but still lacks comprehensiveness by only targeting specific and preconceived microbes $(9,10)$. Unexpected, rare, or novel pathogens may evade detection by serological tests and $16 \mathrm{~S}$ rRNA PCR.

Recently, metagenomic next-generation sequencing (mNGS) has been applied in the etiological diagnosis of infectious diseases as a non-culture-based and universal method (11-13), most frequently used in central nervous system infections (14-16), osteoarticular infections $(17,18)$, skin and soft tissue infections (19) and respiratory infections $(20,21)$. With the capability to detect any potential pathogen in clinical samples, the comprehensive and unbiased nature of mNGS has outperformed serological tests and $16 \mathrm{~S}$ rRNA PCR. However, few studies have reported on the application of mNGS in IE. Jun Cheng et al. compared the sensitivity and specificity of mNGS with culture for identifying IE pathogens but the specific parameters of mNGS results and the significant value of valve mNGS in optimizing IE antibiotic treatment during clinical practice were less discussed (22). To study these issues, our research innovatively adopted valve mNGS to evaluate its etiological diagnostic value in IE. We present the following article in accordance with the MDAR reporting checklist (available at http://dx.doi.org/10.21037/atm-21-2488).

\section{Methods}

\section{Participants}

We retrospectively reviewed 63 patients who underwent valve surgery in Zhongshan Hospital, Fudan University, Shanghai from 1 June 2018 to 30 November 2020, as shown in Figure 1. Among them, 8 patients were admitted with non-infective valvular diseases and their resected valves were subjected to mNGS as negative controls. The remaining 55 patients matched the diagnosis of definite IE according to the modified Duke criteria (23). The inclusion criterion of this study was definite IE according to the modified Duke criteria. The exclusion criterion was valve samples collected during surgery that either were too little to conduct both culture and mNGS or failed to pass quality control of mNGS. A total of 4 IE patients were excluded due to their resected valves being too little to conduct both culture and mNGS. An additional 2 IE patients were excluded because their valve samples failed to pass the quality control of mNGS. Finally, 49 IE patients were investigated in this study. Among them, 28 participants (57.1\%) were blood culture or valve culture positive. Both blood culture and valve culture were negative in the remaining 21 participants (42.9\%). All participants or their legal designees provided written informed consent before participation. Ethical approval was granted by the Ethical Review Committee of Zhongshan Hospital, Fudan University, Shanghai, China (B2017-193R). All procedures performed in this study involving human participants were in accordance with the Declaration of Helsinki (as revised in 2013).

\section{Blood culture and valve culture}

All IE participants underwent blood culture by peripheral venous puncture at least once after admission. Blood samples $(8-10 \mathrm{~mL})$ were injected into aerobic, anaerobic, and fungal blood culture bottles (BD BACTEC ${ }^{\mathrm{TM}}$, Becton, Dickinson, 


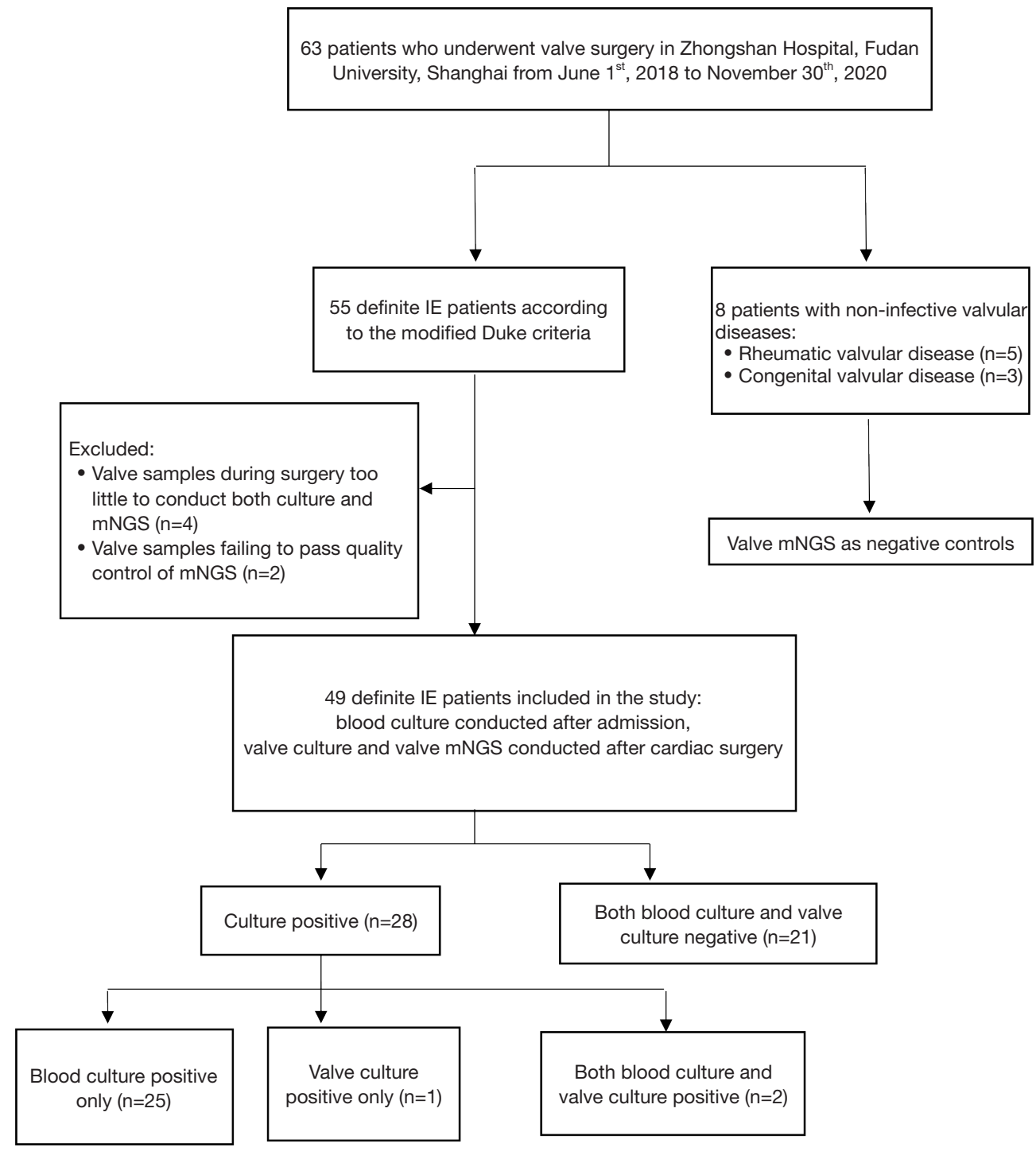

Figure 1 Flowchart of participant recruitment and collection of samples. From a total of 63 patients, 57 were finally included in the analysis. Among them, 8 participants were admitted with non-infective valvular diseases and 49 were definite IE according to the modified Duke criteria. A total of 28 IE patients were blood culture or valve culture positive. Both blood culture and valve culture were negative in the remaining 21 IE participants. mNGS, metagenomic next-generation sequencing; IE, infective endocarditis.

and Co., Franklin Lakes, NJ, USA) and then loaded into an automated continuous monitoring system (BD BACTEC ${ }^{\mathrm{TM}}$, USA) for 7 days. Under sterile conditions, the resected valves were grinded into homogenate. Half of the valve homogenate was cultured onto blood agar plates, chocolate agar plates, and fungal chromogenic plates for at least 14 days. The other half of the valve homogenate was subjected to mNGS. If blood culture or valve culture showed bacterial growth, strain identification was conducted by VITEK MALDI-TOF mass spectrometry (bioMérieux,
Craponne, France).

\section{Valve $m N G S$}

Sample processing, mNGS, and analysis were conducted as described previously. After surgical resection and grinding, deoxyribonucleic acid (DNA) was extracted from the valve homogenate using a TIANamp Micro DNA Kit (DP316, TIANGEN BIOTECH, Beijing, China). With the adapters added overnight, DNA libraries were 
generated via an end-repair method, followed by PCR amplification. Then, an Ion Torrent Proton Sequencer (Life Technologies, Carlsbad, CA, USA) was used for analysis. The quality of the DNA libraries was evaluated by an Agilent 2100 Bioanalyzer (Agilent Technologies, Santa Clara, CA, USA) along with quantitative PCR (qPCR) to measure the adapters before sequencing. Qualified DNA libraries were prepared in a OneTouch system by emulsion PCR and then sequenced on the Ion Torrent Proton (Life Technologies, South San Francisco, CA, USA) sequencing platform. Then, low-quality and short (length $<35 \mathrm{bp}$ ) reads were removed to generate high-quality sequencing data. Human host sequences mapped to the human reference genome (hg19) were subtracted via Burrows-Wheeler Alignment. The remaining nonhuman data were mapped to classification reference databases composed of viruses, bacteria, fungi, and parasites which can be downloaded from the National Center for Biotechnology Information (NCBI, ftp://ftp.ncbi.nlm.nih.gov/genomes/). The RefSeq contains 4,189 whole-genome sequences of viral taxa, 2,328 bacterial genomes, 199 fungi, and 135 parasites related to human infectious diseases.

\section{Criteria for a positive valve $m N G S$ result}

For bacteria, the criteria for a positive value mNGS result were as follows: mNGS identified a bacterium of species level whose coverage rate scored 10 -fold greater than that of any other bacteria according to previous studies $(12,24)$.

Those for fungi were as follows: mNGS identified a fungus of species level whose coverage rate scored 5 -fold greater than that of any other fungi taking account of its low biomass in DNA extraction (25).

Stringent mapped reads number of genera (SMRNG), relative abundance of genera, stringent mapped reads number of species (SMRN), relative abundance of species, and coverage rate were used as the measurement parameters of mNGS results in this study.

\section{Statistical analysis}

Continuous variables were expressed as median (interquartile range) if they followed a non-normal distribution. Comparative analysis between two groups was conducted by Mann-Whitney U-test. Data analysis was performed with the statistical software SPSS version 21.0 (IBM Corp., Chicago, IL, USA). All tests were 2-tailed, and statistical significance was considered at $\mathrm{P}<0.05$.

\section{Results}

\section{Characteristics of the 57 participants}

A total of 57 patients were included in the study, among which 49 were IE and 8 were patients with non-infective valvular diseases as negative controls. Characteristics of the 57 participants are listed in Table 1. Among the negative control participants, the median age was 47 years and $62.5 \%(\mathrm{n}=5)$ were male. Among IE participants, the median age was 50 years; $71.4 \%(n=35)$ were male; $87.8 \%(n=43)$ were native valve endocarditis (NVE); $12.2 \%(\mathrm{n}=6)$ were prosthetic valve endocarditis (PVE); $57.1 \%(\mathrm{n}=28)$ were blood culture or valve culture positive; and 93.9\% $(n=46)$ had used antibiotics before admission.

\section{Valve mNGS results and compositions of pathogens detected by $m N G S$}

The valve mNGS results of the 8 participants with noninfective valvular heart diseases were all negative. Among the 57 IE participants, valve mNGS identified $91.8 \%(\mathrm{n}=45)$ gram positive bacteria, $6.1 \%(\mathrm{n}=3)$ gram negative bacteria, and $2.0 \%(n=1)$ fungus. The detailed valve $\mathrm{mNGS}$ results of the $57 \mathrm{IE}$ patients are displayed in Figure 2. The parameters of IE valve mNGS were as follows: SMRNG: $12,072(3,872$, $141,740)$, relative abundance of genera: $95.5 \%(83.2 \%$, 98.1\%), SMRN: $10,100(1,826,98,866)$, relative abundance of species: $70.1 \%(57.8 \%, 86.5 \%)$, and coverage rate: $26.6 \%$ $(5.7 \%, 65.6 \%)$.

\section{Culture results and the main types of microbes in culture- positive IE}

Among the 28 culture-positive IE participants (P1P28), 25 were blood culture positive solely; 1 was valve culture positive solely; and the remaining 2 participants had both positive and consistent blood culture and valve culture. The main types of microbes in culture-positive IE can be summarized as Gram-positive bacteria $(n=25$, $89.3 \%)$, Gram-negative bacteria $\mathrm{n}=2(7.1 \%)$ and Fungi $\mathrm{n}=1$ (3.6\%), as displayed in Table 2. Microbiological culture identified Abiotrophia defectiva ( $\mathrm{n}=4)$, Streptococcus sanguinis $(\mathrm{n}=3)$, Streptococcus oralis $(\mathrm{n}=3)$, Streptococcus gordonii $(\mathrm{n}=3)$, Streptococcus gallolyticus $(\mathrm{n}=2)$, Staphylococcus lugdunensis $(\mathrm{n}=2)$, Staphylococcus epidermidis $(\mathrm{n}=2)$, Granulicatella adiacens $(\mathrm{n}=2)$, Streptococcus salivarius $(\mathrm{n}=1)$, Streptococcus mitis $(\mathrm{n}=1)$, Enterococcus faecalis $(\mathrm{n}=1)$, Erysipelothrix rbusiopathiae $(\mathrm{n}=1)$, Cardiobacterium hominis $(\mathrm{n}=1)$, Aggregatibacter segnis $(\mathrm{n}=1)$, 
Table 1 Characteristics of the 57 participants

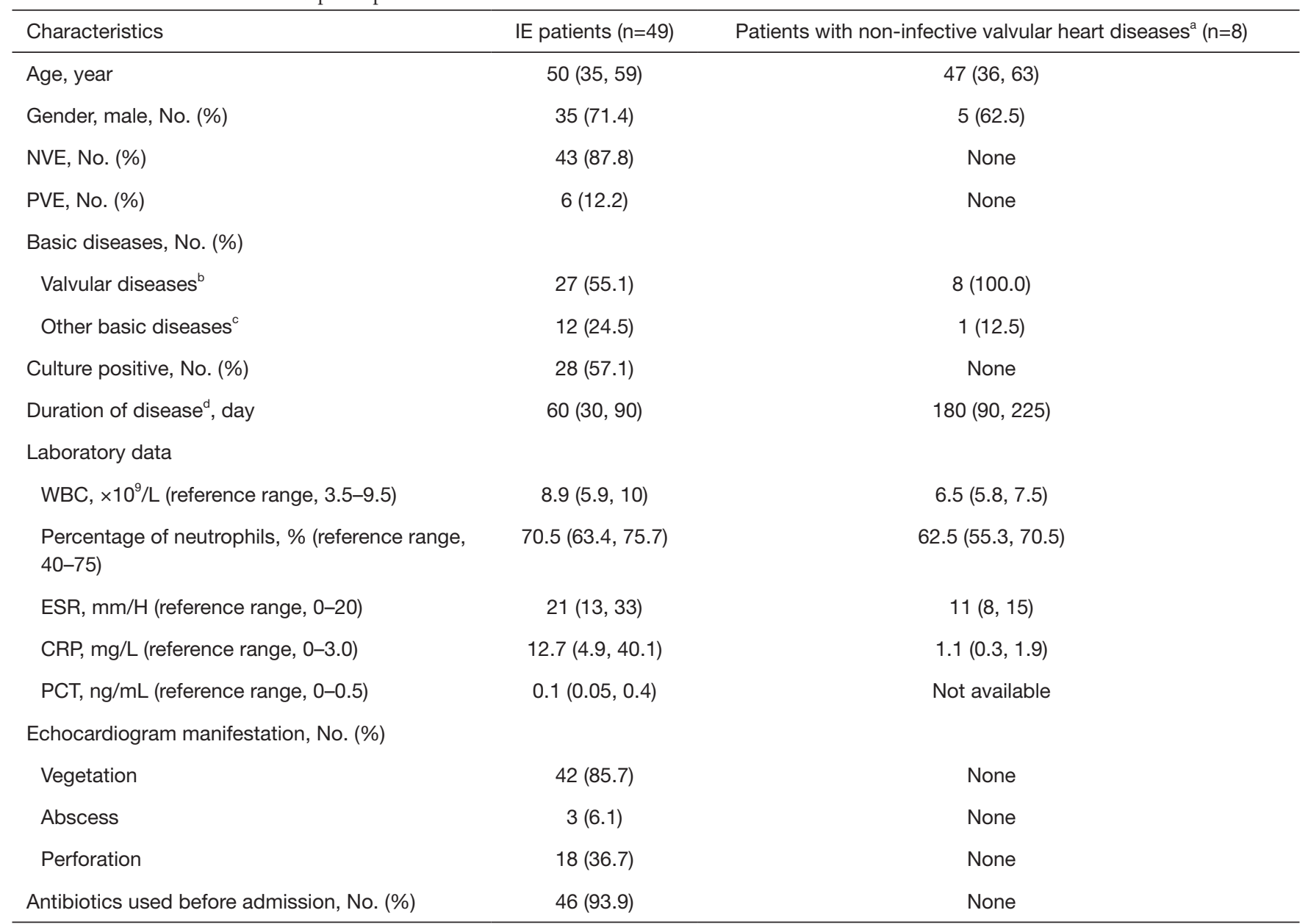

${ }^{a}$, including rheumatic valvular disease $(n=5)$ and congenital valvular disease $(n=3) ;{ }^{b}$, including rheumatic valvular disease, congenital valvular disease, and degenerative valvular disease; ${ }^{c}$, including diabetes mellitus, chronic renal failure, liver cirrhosis, malignant tumor, long term administration of glucocorticoid, or other kinds of immunocompromised conditions; ${ }^{d}$, from onset of fever, chest tightness, shortness of breath, or other symptoms to admission. NVE, native valve endocarditis; PVE, prosthetic valve endocarditis; ESR, erythrocyte sedimentation rate; CRP, C-reactive protein; PCT, procalcitonin.

and Candida parapsilosis $(\mathrm{n}=1)$. Representative result images of MALDI-TOF mass spectrometry of IE culture isolates are displayed in Figure 3.

\section{Reliability of valve mNGS in the etiological diagnosis of IE}

For the 28 culture-positive IE participants, the valve mNGS results were exactly consistent with microbiological culture results on both genera and species level. With microbiological culture results set as the gold standard, the sensitivity and specificity of valve mNGS in the etiological diagnosis of IE were both $100 \%$.

\section{Performance of valve mNGS in culture-negative IE patients}

The positive detection rate of pathogens by valve mNGS was $100 \%$ for the 21 culture-negative IE participants (P29-P49). Valve mNGS identified Streptococcus sanguinis $(\mathrm{n}=3)$, Streptococcus oralis $(\mathrm{n}=3)$, Streptococcus salivarius $(\mathrm{n}=2)$, Streptococcus dysgalactiae $(\mathrm{n}=2)$, Staphylococcus lugdunensis $(\mathrm{n}=2)$, Streptococcus gordonii $(\mathrm{n}=1)$, Streptococcus sinensis $(\mathrm{n}=1)$, Streptococcus vestibularis $(\mathrm{n}=1)$, Staphylococcus epidermidis $(\mathrm{n}=1)$, Staphylococcus capitis $(\mathrm{n}=1)$, Staphylococcus haemolyticus $(\mathrm{n}=1)$, Staphylococcus bominis $(\mathrm{n}=1)$, Gemella haemolysans $(\mathrm{n}=1)$, and 


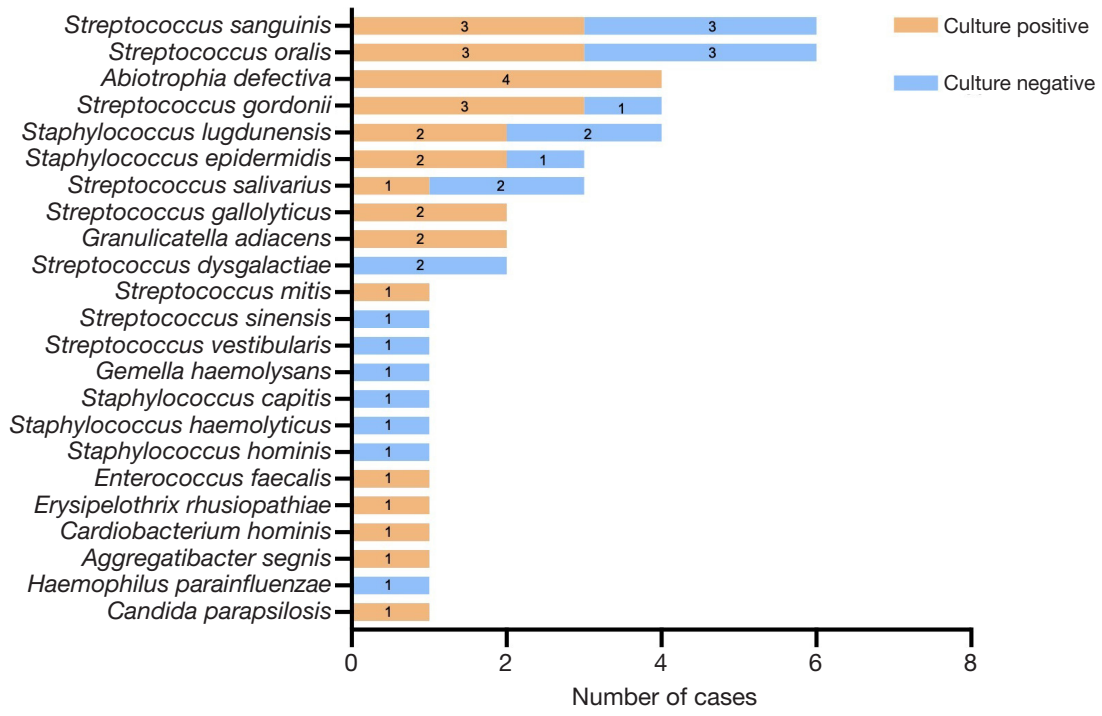

Figure 2 Pathogens of IE detected by valve mNGS and number of cases. mNGS, metagenomic next-generation sequencing; IE, infective endocarditis.

Table 2 The main types of microbes in culture-positive IE

\begin{tabular}{lcc}
\hline Microbes in culture-positive IE & $\begin{array}{c}\text { Number of } \\
\text { cases }(\mathrm{n}=28)\end{array}$ & $\begin{array}{c}\text { Constituent } \\
\text { ratios (\%) }\end{array}$ \\
\hline Gram-positive bacteria & 25 & 89.3 \\
Streptococcus spp. & 13 & 46.4 \\
Staphylococcus spp. & 4 & 14.3 \\
Other Gram-positive bacteria & 8 & 28.6 \\
Gram-negative bacteria & 2 & 7.1 \\
Fungi & 1 & 3.6 \\
\hline
\end{tabular}

IE, infective endocarditis.

Haemophilus parainfluenzae $(\mathrm{n}=1)$.

\section{The differences of valve mNGS results between culture- positive IE and culture-negative IE participants}

The SMRNG, relative abundance of genera, SMRN, relative abundance of species, and coverage rate of valve mNGS results were significantly higher in culture-positive IE participants than in those with culture-negative IE $(\mathrm{P}<0.05)$, as shown in Table 3.

\section{Valve mNGS optimizing antibiotic treatment in culture-} negative IE patients

All 21 culture-negative IE participants received empirical antibiotic treatment, usually broad-spectrum antibiotics such as carbapenems, vancomycin, and daptomycin. After identifying pathogens by valve mNGS, antibiotic treatment of 6 culture-negative IE participants was adjusted and deescalated to cephalosporin and aminoglycosides. Extensive antibiotic abuse was avoided. Details of antibiotic treatment adjustment are listed in Table 4.

\section{Discussion}

In this study, valve mNGS was confirmed as a reliable method to identify IE pathogens by its complete consistency with microbiological culture. Compared to culture, valve mNGS has higher sensitivity in the etiological diagnosis of IE, providing important information for targeted antibiotic treatment after cardiac surgery, especially for culturenegative IE patients.

The treatment for IE includes medical therapy and valvular surgery. Antibiotic therapy lasts from 2 weeks for penicillin-susceptible streptococcal NVE to 6 weeks for PVE. Antibiotic choice is made mainly according to the causative pathogen, demanding a rapid and precise etiological diagnosis. Valvular surgery is indicated under the conditions of heart failure, uncontrolled infection or prevention of embolism. The aim of valvular surgery is to resect all infected tissues and to restore normal valve function (2).

A precise etiological diagnosis is the cornerstone of 

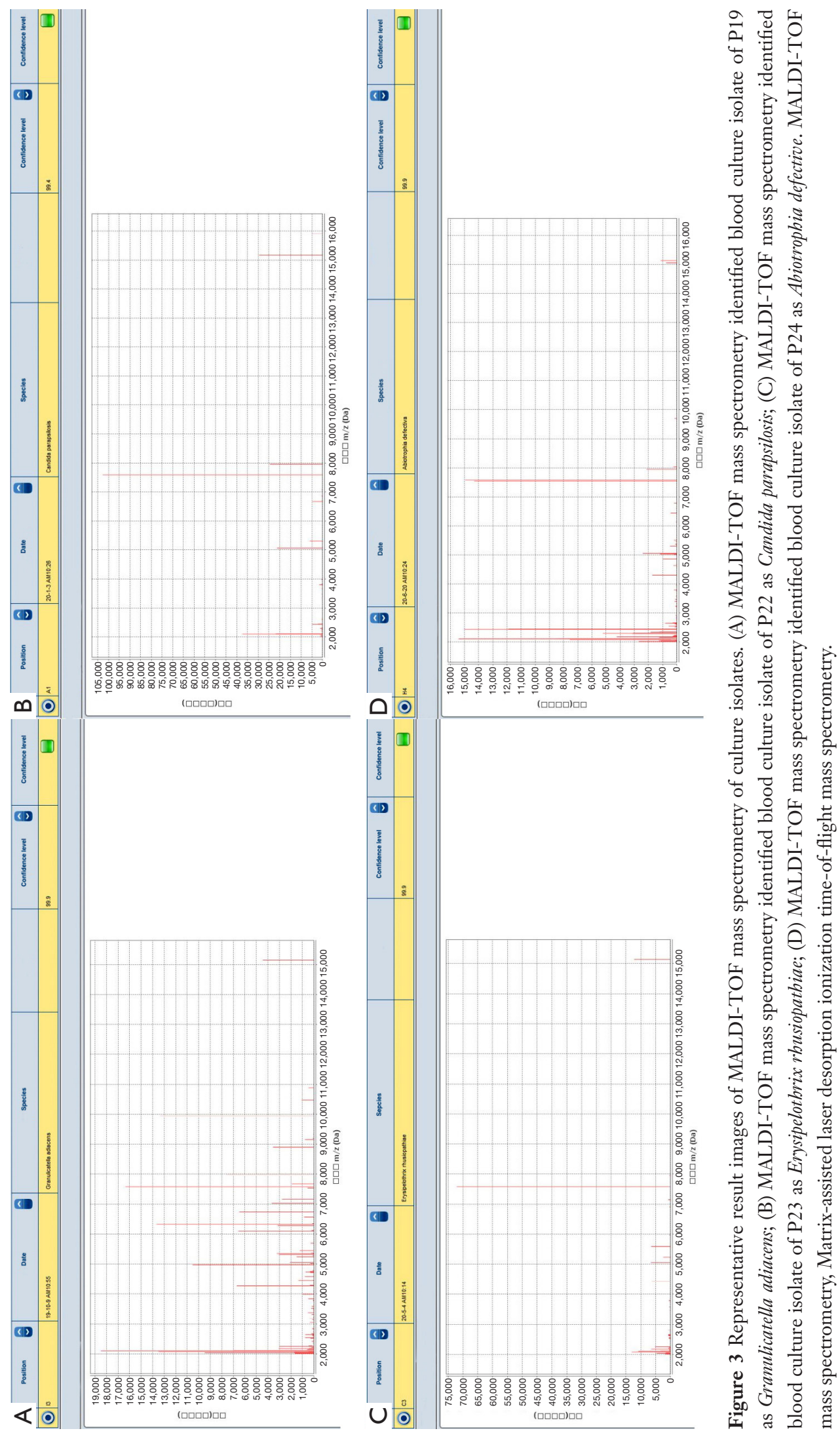
Table 3 The differences of valve mNGS results between culture-positive IE and culture-negative IE patients

\begin{tabular}{lccc}
\hline Parameters of mNGS results & Culture-positive IE & Culture-negative IE & P value \\
\hline SMRNG & $32,209(9,518,179,897)$ & $7,032(1,278,24,719)$ & 0.0199 \\
Relative abundance of genera & $97.4 \%(94.1 \%, 98.2 \%)$ & $84.8 \%(68.7 \%, 96.8 \%)$ & $3,480(815,15,005)$ \\
SMRN & $28,596(5,250,157,095)$ & 0.0063 \\
Relative abundance of species & $75.4 \%(66.0 \%, 95.7 \%)$ & $63.9 \%(52.2 \%, 76.1 \%)$ & 0.0161 \\
Coverage rate & $41.5 \%(16.7 \%, 75.6 \%)$ & $14.2 \%(3.4 \%, 47.8 \%)$ & 0.043 \\
\hline
\end{tabular}

mNGS, metagenomic next-generation sequencing; IE, infective endocarditis; SMRNG, stringent mapped reads number of genera; SMRN, stringent mapped reads number.

Table 4 Valve mNGS optimizing antibiotic treatment in culture-negative IE patients

\begin{tabular}{lllll}
\hline Patient ID & Type of IE & $\begin{array}{l}\text { Pathogens identified by valve } \\
\text { mNGS }\end{array}$ & Initial antibiotic regimen before mNGS & $\begin{array}{l}\text { Adjusted antibiotic regimen after } \\
\text { mNGS }\end{array}$ \\
\hline 30 & NVE & Streptococcus oralis & Daptomycin & Ceftriaxone \\
34 & NVE & Streptococcus oralis & Vancomycin + meropenem & Ceftriaxone + Amikacin \\
35 & NVE & Streptococcus oralis & Vancomycin + meropenem & Cefuroxime \\
38 & NVE & Haemophilus parainfluenzae & Daptomycin & Ceftriaxone \\
40 & NVE & Streptococcus sanguinis & Vancomycin + imipenem cilastatin & Ceftriaxone + Amikacin \\
42 & PVE & Streptococcus salivarius & Vancomycin + meropenem & Ceftriaxone + Amikacin \\
\hline
\end{tabular}

mNGS, metagenomic next-generation sequencing; IE, infective endocarditis; NVE, native valve endocarditis; PVE, prosthetic valve endocarditis.

proper antibiotic treatment of IE (4). However, in the real world most patients have already received antibiotic treatment before admission, usually leading to sterile microbiological cultures (8). In this study, 93.9\% IE patients had used antibiotics before admission, and the remaining $6.1 \%$ of IE patients also received antibiotic treatment after admission prior to cardiac surgery. Meanwhile, the duration of disease for some IE participants was very long. In this study, 8 IE participants (16.3\%) had experienced recurrent fever for more than 180 days and their white blood cell count as well as serum inflammatory markers had almost returned to normal range at admission, indicating the subacute course of IE. Prior antibiotic exposure and the subacute course of IE significantly reduced the positive rate of culture. As a culture-independent method, valve mNGS eliminated such a quandry, identifying pathogens even for patients with a subacute course and multiple antibiotic exposure.

For IE patients with an acute course whose temperature, white blood cell count and serum inflammatory markers were rather high, blood mNGS might be a promising etiological diagnostic technology. However, for IE patients with a subacute course whose temperature, white blood cell count and serum inflammatory markers had almost returned to normal range, the positive detection rate of pathogens by blood mNGS was lower than valve mNGS. The current cost of mNGS in Shanghai is 3,000 RMB per sample. Therefore, for IE patients with a subacute course, we assume valve mNGS more suitable than blood mNGS if the patient's economic condition is not sufficient to conduct both. This study included IE cases caused by fastidious bacteria such as Cardiobacterium hominis (P10), Aggregatibacter segnis (P18), Haemophilus parainfluenzae (P38), Abiotrophia defective (P14, P16, P17, P24), and Granulicatella adiacens (P15, P19). Such fastidious bacteria grow relatively slowly and require specific media and conditions, resulting in further reduction of the positive rate of culture. The incubation time of these fastidious bacteria frequently extends to at least 7 days, leading to delay of etiological diagnosis and antibiotic adjustment. Valve mNGS possesses a significantly higher positive detection rate and shortens the turnaround time to $48 \mathrm{~h}$, thus offering 
prompt guidance for antibiotic adjustment and improving the prognosis of IE.

As a comprehensive and unbiased method, mNGS screens for all the potential pathogens from clinical specimens, assisting to identify unexpected or novel organisms. Regarding comprehensiveness, mNGS outperforms other non-culture-based methods such as serological testing or $16 \mathrm{~S}$ rRNA PCR which can only target specific microbes (12). 16S rRNA PCR can only detect preconceived pathogens, limiting the diagnostic value in IE caused by rare or emerging pathogens. However, the comprehensive nature of mNGS allows us to detect any potential pathogens, meeting the challenges of rare and emerging microbes.

In this study we discovered that SMRNG, relative abundance of genera, SMRN, relative abundance of species, and coverage rate of valve mNGS were significantly higher in culture-positive IE patients than in culture-negative IE patients. This phenomenon is probably associated with the prior effective antibiotic treatment in culture-negative IE patients. Effective antibiotic treatment before surgery leads to sterile microbiological cultures as well as reduction of pathogen nucleic acid load detected by valve mNGS. This phenomenon also indicates the important value of valve mNGS with high sensitivity in the etiological diagnosis of IE.

Among the 21 culture-negative IE participants in this study, 9 (42.9\%) received empirical broad-spectrum antibiotics such as carbapenems, vancomycin, and daptomycin after admission and should have instead received de-escalated antibiotic treatment after pathogen identification by valve mNGS. However, considering the severe situation of multidrug resistant bacteria infection in the intensive care unit after surgery and the risk of nosocomial infection, 3 participants $(14.3 \%)$ failed to receive promptly de-escalated antibiotic treatment. The remaining 6 participants $(28.6 \%)$ had their antibiotics adjusted to cephalosporin and aminoglycosides after positive valve $\mathrm{mNGS}$ results. This finding indicated the value of valve $\mathrm{mNGS}$ in guiding targeted antibiotic treatment instead of empirical antibiotic treatment and decreasing unnecessary usage of broad-spectrum antibiotics.

There were some limitations in this study. First, the number of participants was not enough to compare the parameters of valve mNGS results between different pathogens. Neither could we divide the participants into subgroups according to demographic characteristics. Second, this was a single-center retrospective study and participant selection bias may have existed. Third, we did not detect antibiotic resistance genes in this study.
In conclusion, valve mNGS has great value in the etiological diagnosis of IE. Our research showed the reliability and sensitivity of valve mNGS in clinical practice to IE patients. Valve mNGS provides important information for precise antibiotic treatment of culturenegative IE patients after cardiac surgery.

\section{Acknowledgments}

The authors appreciate all the clinicians, microbiologists, and pathologists who have assisted this research.

Funding: This study was supported by the Clinical Research Funds of Zhongshan Hospital, Fudan University (2018ZSLC06).

\section{Footnote}

Reporting Checklist: The authors have completed the MDAR reporting checklist. Available at http://dx.doi.org/10.21037/ atm-21-2488

Data Sharing Statement: Available at http://dx.doi. org/10.21037/atm-21-2488

Conflicts of Interest: All authors have completed the ICMJE uniform disclosure form (available at http://dx.doi. org/10.21037/atm-21-2488). The authors have no conflicts of interest to declare.

Ethical Statement: The authors are accountable for all aspects of the work in ensuring that questions related to the accuracy or integrity of any part of the work are appropriately investigated and resolved. All participants or their legal designees provided written informed consent before participation. Ethical approval was granted by the Ethical Review Committee of Zhongshan Hospital, Fudan University, Shanghai, China (B2017-193R). All procedures performed in this study involving human participants were in accordance with the Declaration of Helsinki (as revised in 2013).

Open Access Statement: This is an Open Access article distributed in accordance with the Creative Commons Attribution-NonCommercial-NoDerivs 4.0 International License (CC BY-NC-ND 4.0), which permits the noncommercial replication and distribution of the article with the strict proviso that no changes or edits are made and the original work is properly cited (including links to both the 


\section{Page 10 of 11}

formal publication through the relevant DOI and the license). See: https://creativecommons.org/licenses/by-nc-nd/4.0/.

\section{References}

1. Habib G, Lancellotti P, Antunes MJ, et al. 2015 ESC Guidelines for the management of infective endocarditis: The Task Force for the Management of Infective Endocarditis of the European Society of Cardiology (ESC). Endorsed by: European Association for Cardio-Thoracic Surgery (EACTS), the European Association of Nuclear Medicine (EANM). Eur Heart J 2015;36:3075-128.

2. Iung B, Duval X. Infective endocarditis: innovations in the management of an old disease. Nat Rev Cardiol 2019;16:623-35.

3. Cahill TJ, Baddour LM, Habib G, et al. Challenges in Infective Endocarditis. J Am Coll Cardiol 2017;69:325-44.

4. Subedi S, Jennings Z, Chen SC. Laboratory approach to the diagnosis of culture-negative infective endocarditis. Heart Lung Circ 2017;26:763-71.

5. Lützen L, Olesen B, Voldstedlund M, et al. Incidence of HACEK bacteraemia in Denmark: A 6-year populationbased study. Int J Infect Dis 2018;68:83-7.

6. Okaro U, Addisu A, Casanas B, et al. Bartonella Species, an Emerging Cause of Blood-Culture-Negative Endocarditis. Clin Microbiol Rev 2017;30:709-46.

7. Sullivan A, Shrestha P, Basnet $\mathrm{S}$, et al. A rare case of Whipple's disease with endocarditis in a patient with dextrocardia. SAGE Open Med Case Rep 2020;8:2050313X20936952.

8. Fournier PE, Thuny F, Richet H, et al. Comprehensive Diagnostic Strategy for Blood Culture-Negative Endocarditis: A Prospective Study of 819 New Cases. Clin Infect Dis 2010;51:131-40.

9. Godfrey R, Curtis S, Schilling WH, et al. Blood culture negative endocarditis in the modern era of $16 \mathrm{~S} \mathrm{rRNA}$ sequencing. Clin Med (Lond) 2020;20:412-6.

10. Fournier PE, Gouriet F, Casalta JP, et al. Blood culturenegative endocarditis. Medicine 2017;96:e8392.

11. Gu W, Deng X, Lee M, et al. Rapid pathogen detection by metagenomic next-generation sequencing of infected body fluids. Nat Med 2021;27:115-24.

12. Miao Q, Ma Y, Wang Q, et al. Microbiological Diagnostic Performance of Metagenomic Next-generation Sequencing When Applied to Clinical Practice. Clin Infect Dis 2018;67:S231-40.

13. Zhou X, Wu H, Ruan Q, et al. Clinical Evaluation of

\section{Cai et al. Metagenomic sequencing in infective endocarditis}

Diagnosis Efficacy of Active Mycobacterium tuberculosis Complex Infection via Metagenomic Next-Generation Sequencing of Direct Clinical Samples. Front Cell Infect Microbiol 2019;9:351.

14. Qian L, Shi Y, Li F, et al. Metagenomic Next-Generation Sequencing of Cerebrospinal Fluid for the Diagnosis of External Ventricular and Lumbar DrainageAssociated Ventriculitis and Meningitis. Front Microbiol 2020;11:596175.

15. Xing XW, Zhang JT, Ma YB, et al. Metagenomic NextGeneration Sequencing for Diagnosis of Infectious Encephalitis and Meningitis: A Large, Prospective Case Series of 213 Patients. Front Cell Infect Microbiol 2020;10:88.

16. Tsamis KI, Sakkas H, Giannakis A, et al. Evaluating Infectious, Neoplastic, Immunological, and Degenerative Diseases of the Central Nervous System with Cerebrospinal Fluid-Based Next-Generation Sequencing. Mol Diagn Ther 2021;25:207-29.

17. Huang ZD, Zhang ZJ, Yang B, et al. Pathogenic Detection by Metagenomic Next-Generation Sequencing in Osteoarticular Infections. Front Cell Infect Microbiol 2020;10:471.

18. Cai Y, Fang X, Chen Y, et al. Metagenomic next generation sequencing improves diagnosis of prosthetic joint infection by detecting the presence of bacteria in periprosthetic tissues. Int J Infect Dis 2020;96:573-8.

19. Wang Q, Miao Q, Pan J, et al. The clinical value of metagenomic next-generation sequencing in the microbiological diagnosis of skin and soft tissue infections. Int J Infect Dis 2020;100:414-20.

20. van Boheemen S, van Rijn AL, Pappas N, et al. Retrospective Validation of a Metagenomic Sequencing Protocol for Combined Detection of RNA and DNA Viruses Using Respiratory Samples from Pediatric Patients. J Mol Diagn 2020;22:196-207.

21. Shi CL, Han P, Tang PJ, et al. Clinical metagenomic sequencing for diagnosis of pulmonary tuberculosis. J Infect 2020;81:567-74.

22. Cheng J, Hu H, Fang W, et al. Detection of pathogens from resected heart valves of patients with infective endocarditis by next-generation sequencing. Int $\mathrm{J}$ Infect Dis 2019;83:148-53.

23. Li JS, Sexton DJ, Mick N, et al. Proposed Modifications to the Duke Criteria for the Diagnosis of Infective Endocarditis. Clin Infect Dis 2000;30:633-8.

24. Langelier C, Zinter MS, Kalantar K, et al. Metagenomic Sequencing Detects Respiratory Pathogens in 
Hematopoietic Cellular Transplant Patients. Am J Respir Crit Care Med 2018;197:524-8.

25. Schlaberg R, Chiu CY, Miller S, et al. Validation of Metagenomic Next-Generation Sequencing Tests for

Cite this article as: Cai S, Yang Y, Pan J, Miao Q, Jin W, Ma Y, Zhou C, Gao X, Wang C, Hu B. The clinical value of valve metagenomic next-generation sequencing when applied to the etiological diagnosis of infective endocarditis. Ann Transl Med 2021;9(19):1490. doi: 10.21037/atm-21-2488
Universal Pathogen Detection. Arch Pathol Lab Med 2017;141:776-86.

(English Language Editor: J. Jones) 